\title{
THE GEOMETRIC CONFIGURATION DEFINED BY A SPECIAL ALGEBRAIC RELATION OF GENUS FOUR*
}

\author{
BY \\ FRANCES HARSHBARGER
}

INTRODUCTION

The double binary form, $(\alpha t)^{3}(a \tau)^{3}$, of third order in digredient variables $t$ and $\tau$, when equated to zero, defines a space curve of the sixth order on a quadric surface with generators $t$ and $\tau$. This space curve is the complete intersection of the quadric surface and a cubic surface and hence is of genus four. The geometric theory connected with a general $(3,3)$ form has been discussed by Coble. $\dagger$ He introduced the form, with the interpretation also used by H. S. White, $\ddagger$ that is, as the incidence condition of a point of one space cubic curve and a plane of a second space cubic curve.

It is the purpose of this paper to discuss the special form

$$
F=t_{1}^{3} \tau_{1}^{2} \tau_{2}+t_{1}^{2} t_{2} \tau_{2}^{3}+t_{1} t_{2}^{2} \tau_{1}^{8}-t_{2}^{3} \tau_{1} \tau_{2}^{2} .
$$

This form was first investigated by Gordan, $\S$ who followed Klein in considering its relation to the solution of the equation of the fifth degree and to the icosahedral equation.

When the equation of the fifth degree has the form

$$
x^{5}+5 \alpha x^{2}+5 \beta x+\gamma=0,
$$

then $\sum_{i=0}^{i=4} x_{i}=0$ and $\sum_{i=0}^{i=4} x_{i}{ }^{2}=0$, and the $x_{i}$ may be taken as the pentahedral coördinates of a point in space and $\sum_{i=0}^{i=4} x_{i}{ }^{2}=0$ is a quadric surface unaltered by the group of the 120 permutations of the $x_{i}$ 's. Klein $\|$ sets

$$
p_{i}=x_{0}+\epsilon^{i} x_{1}+\epsilon^{2 i} x_{2}+\epsilon^{3 i} x_{3}+\epsilon^{4 i} x_{4}, \epsilon=e^{2 \pi i / 5}(i=1,2,3,4),
$$

or

$$
5 x_{\nu}=\epsilon^{4 \nu} p_{1}+\epsilon^{3 \nu} p_{2}+\epsilon^{2 \nu} p_{3}+\epsilon^{\nu} p_{4} \quad(\nu=0,1,2,3,4),
$$

* Presented to the Society, February 28, 1931; received by the editors February 4, 1931.

$\dagger$ Coble, Geometric aspects of the abelian modular functions of genus four, American Journal of Mathematics, vol. 46 (1924), pp. 143-192; Geometric aspects of the abelian modular functions of genus four, Part II, American Journal of Mathematics, vol. 51 (1929), pp. 495-514.

$\ddagger$ White, $A$ variable system of sevens on two twisted cubic curves, Proceedings of the National Academy of Sciences, vol. 2 (1916), p. 337.

$\S$ Gordan, Ueber die Aufösung der Gleichungen fünften Grades, Erlangen Physikalisch-Medicinische Societät, Sitzungsberichte, vol. 9 (1877), pp. 183-186; Ueber die Auflösung der Gleichungen vom fünften Grade, Mathematische Annalen, vol. 13 (1878), pp. 375-404.

|| Klein, Vorlesungen über das Ikosaeder, Leipzig, 1884, p. 187. 
and $t_{1} / t_{2}=-p_{1} / p_{2}=p_{3} / p_{4}, \tau_{1} / \tau_{2}=p_{1} / p_{3}=-p_{2} / p_{4}$. Then $25 \sum_{\nu=0}^{v=4} x_{\nu}^{2}=10\left(p_{1} p_{4}\right.$ $\left.+p_{2} p_{3}\right)$. That is, the quadric $\sum_{v=0}^{v=4} x_{\nu}^{2}=0$ becomes the quadric $p_{1} p_{4}+p_{2} p_{3}=0$ with generators $t=t_{1}: t_{2}$ and $\tau=\tau_{1}: \tau_{2}$. If then

$$
p_{1}=-5 t_{1} \tau_{1}, \quad p_{2}=5 t_{2} \tau_{1}, \quad p_{3}=-5 t_{1} \tau_{2}, \quad p_{4}=-5 t_{2} \tau_{2},
$$

$\alpha=-\sum_{i=0}^{i=4} x_{i}{ }^{3} / 15$ is in terms of $t$ and $\tau$ equal to

$$
F=t_{1}^{3} \tau_{1}^{2} \tau_{2}+t_{1}^{2} t_{2} \tau_{2}^{3}+t_{1} t_{2}^{2} \tau_{1}^{3}-t_{2}^{3} \tau_{1} \tau_{2}^{2}
$$

Gordan* computed the complete system of thirty-six covariants for the form $F$ as a form in digredient variables.

In an article referred to above, $\uparrow$ Coble derives the complete system of covariants of degrees one, two, and three for the general $(3,3)$ form. He then proceeds to the figure of two cubic curves in space. The net of quadrics and the net of quadric envelopes on one space cubic curve cut out involutions $I_{2}^{6}$ on the other. The four $I_{2}^{6}$ 's thus determined can be interpreted as four rational planar sextics. Any one of the four determines the figure of the two cubic curves in space. Conner $\ddagger$ and Coble $\S$ also discuss the quartic Jacobian surface and the quartic surface (the symmetroid of Cayley) which are birationally related to each other and to the figure of two cubic space curves.

It is the purpose of this paper to examine this geometric situation for the special form $F$ which admits a $G_{120}$. The geometric figures determined by $F$ are therefore also invariant under a group. In particular the rational sextics admit an icosahedral $G_{60}$ and from this point of view have been discussed by Klein, $\|$ and Winger. $\|$ The types of quartic surfaces, of rational space sextic curves, and of pairs of space cubic curves considered here, have not been examined heretofore. They define interesting configurations with remarkably simple geometric behavior and a correspondingly simple algebra and thus are useful as a check on the general case.

Part of the planar configuration can be obtained by the projection of a certain family of elliptic norm curves. This procedure is developed in I. It af-

* Mathematische Annalen, loc. cit.

† American Journal of Mathematics, vol. 46, loc. cit.

¥ J. R. Conner, The rational sextic curve, and the Cayley symmetroid, American Journal of Mathematics, vol. 37 (1915), pp. 29-42, \$4.

$\$$ American Journal of Mathematics, vol. 46, loc. cit., $\$ 10$.

|| Klein, Weitere Untersuchungen über das Ikosaeder, Mathematische Annalen, vol. 12 (1877), pp. 503-560; also Vorlesungen über das Ikosaeder.

I R. M. Winger, Self-projective rational sextics, American Journal of Mathematics, vol. 38 (1916), pp. 45-56; On the invariants of the ternary icosahedral group, Mathematische Annalen, vol. 93 (1925), pp. 210-216. 
fords a simple introduction of the modular functions into the theory of the quintic equation as given by Klein.*

I. The $(3,3)$ Form AND the Elliptic QUiNTIC in $S_{4}$

The $(3,3)$ form (1) may be introduced by means of the elliptic quintic $E^{b}$ in $S_{4}$. Klein and Fricke $\nmid$ have discussed the general case $E^{n}$ in $S_{n-1}$ and Bianchił and Miss B. I. Miller $\S$ have considered the special case $n=5$ in some detail.

The parametric equations of $E^{5}$ in $S_{4}$ as given by Klein and Fricke\| become

$$
\begin{aligned}
& X_{\alpha}(u)=a_{\alpha} \cdot \exp \left[-\left(\left(\bar{\eta}_{1}-5 \eta_{1}\right) /\left(2 \omega_{1}\right)\right) u^{2}+(\alpha / 5) \bar{\eta}_{1}\left(u-\alpha \omega_{1} / 10\right)\right] \\
& \cdot \sigma\left(u-\frac{\alpha}{5} \omega_{1} \mid \omega_{1}, \frac{\omega_{2}}{5}\right) \quad(\alpha=0,1,2,3,4) .
\end{aligned}
$$

The five linearly independent quadrics which contain and completely define $E^{5}$ are

$$
Q_{i}=\alpha_{0} X_{i}{ }^{2}+\alpha_{1} X_{i+1} X_{i-1}+\alpha_{2} X_{i+2} X_{i+3}=0 \quad(i=0,1,2,3,4),
$$

where the ratios of the $\alpha$ 's are modular functions. Then the linear system of quadrics on $E^{5}$ is

$$
(L Q)=\sum_{i=0}^{i-4} L_{i} Q_{i}=0
$$

Make the following transformation of variables instead of that used by Miss Miller:**

$$
\begin{array}{lll}
Y_{0}=2 X_{0}, & \zeta_{0}=L_{0}, & \\
Y_{1}=X_{1}+X_{4}, Z_{1}=X_{1}-X_{4}, & \zeta_{1}=L_{1}+L_{4}, \quad \theta_{1}=L_{1}-L_{4}, \\
Y_{2}=X_{2}+X_{3}, Z_{2}=X_{2}-X_{3}, & \zeta_{2}=L_{2}+L_{3}, \quad \theta_{2}=L_{2}-L_{3} .
\end{array}
$$

In terms of the variables $Y$ and $Z$, the linear system of quadrics becomes

$$
4(L Q)=F_{1}+F_{2}+F_{3}=0,
$$

* Vorlesungen über das Ikosaeder.

$\dagger$ Klein-Fricke, Theorie der elliptischen Modulfunktionen, Leipzig, 1890-92..

$\ddagger$ Bianchi, Ueber die Normalformen dritter und fünfter Stufe des elliptischen Integrals erster Gattung, Mathematische Annalen, vol. 17 (1880), pp. 234-262.

\$ B. I. Miller, A new canonical form of the elliptic integral, these Transactions, vol. 17 (1916), pp. 259-283.

|l Loc. cit., p. 263.

II Bianchi, loc. cit., p. 251.

** Loc. cit., p. 278. 
where

$$
\begin{array}{lr}
F_{1}=\left\{\sum \alpha_{i} \alpha_{j} Y_{j+i} Y_{j-i}\right\}_{5} & (i, j=0,1,2) ; \\
F_{2}=\sum(\alpha \zeta)_{i j} Z_{j+i} Z_{j-i} & (i, j=0,1,2, j>i) ; \\
F_{3}=\sum \theta_{i} \alpha_{i}\left(Y_{i-j} Z_{i+j}+Y_{i+j} Z_{i-j}\right) & (i=1,2, j=0,1,2) .
\end{array}
$$

In $F_{1},\{\}_{\zeta}$ indicates polarization once with respect to $\zeta$ and $(\alpha \zeta)_{i j}$ in $F_{2}$ is written for $\alpha_{i} \zeta_{j}-\alpha_{j} \zeta_{i}$. The quadrics on $E^{5}$ are the coefficients of $\zeta$ and $\theta$. Explicitly, they may be written

(3)

\begin{tabular}{c|ccc|ccc}
$\zeta_{0}$ & $\alpha_{0}$ & $\alpha_{1}$ & $\alpha_{2}$ & $\alpha_{0}$ & $\alpha_{1}$ & $\alpha_{2}$ \\
\cline { 2 - 6 }$\zeta_{1}$ & $Y_{0}^{2}$ & $Y_{1}^{2}$ & $Y_{2}^{2}$ & & $-Z_{1}^{2}$ & $-Z_{2}^{2}$ \\
$\zeta_{2}$ & $Y_{1}^{2}$ & $Y_{0} Y_{2}$ & $Y_{1} Y_{2}$ & $Z_{1}^{2}$ & & $Z_{1} Z_{2}$ \\
$\theta_{1}$ & $Y_{1} Y_{2}$ & $Y_{0} Y_{1}$ \\
$\theta_{2}$ & $2 Y_{1} Z_{1}$ & $Y_{0} Z_{2}$ & $-Y_{1} Z_{2}-Y_{2} Z_{1}$ \\
$\theta_{2}^{2}$ & $-Z_{1} Z_{2}$ \\
\hline & $2 Y_{2} Z_{2}$ & $-Y_{1} Z_{2}+Y_{2} Z_{1}$ & $-Y_{0} Z_{1}$ & & &
\end{tabular}

The $E^{5}$ is invariant under a $G_{50}$ generated by

$$
u^{\prime}=-u, u^{\prime}=u+\frac{\omega_{1}}{5}, \quad u^{\prime}=u+\frac{\omega_{2}}{5}
$$

or in terms of the coördinates $X$,

$$
\pi_{1} X_{\alpha}^{\prime}=X_{n-\alpha}, \pi_{2} X_{\alpha}^{\prime}=X_{\alpha-1}, \pi_{3} X_{\alpha}^{\prime}=e^{2 \pi i \alpha / 5} X_{\alpha} .
$$

The family of $E^{5}$ 's is invariant under a group $G_{50.60}$ generated by (5) and

$$
S:\left\{\begin{array}{l}
\omega_{1}^{\prime}=\omega_{1}+\omega_{2}, \\
\omega_{2}^{\prime}=\omega_{2},
\end{array} \quad \text { and } T:\left\{\begin{array}{l}
\omega_{1}^{\prime}=-\omega_{2}, \\
\omega_{2}^{\prime}=\omega_{1},
\end{array}\right.\right.
$$

or, in terms of the coördinates $X, \dagger$

$$
S: \pi X_{\alpha}^{\prime}=\epsilon^{\alpha(\alpha-5) / 2} X_{\alpha} \text {, and } T: \pi X_{\alpha}^{\prime}=\sum_{\beta} \epsilon^{-\alpha \beta} X_{\beta} .
$$

The $G_{50}$ is an invariant subgroup of the $G_{50.60}$.

It is obvious that there are two skew spaces which are pointwise invariant under the involution $u^{\prime}=-u$, that is, the line $S_{1}(Z)$ for which the $Y^{\prime}$ 's are zero and the plane $S_{2}(Y)$ for which the $Z$ 's are zero. The factor group $G_{60}$ of of $G_{50}$ in $G_{50.60}$ is then represented as a collineation group in the spaces $S_{1}(Z)$ and $S_{2}(Y)$ and hence must be isomorphic with the icosahedral group. For the

* Klein-Fricke, loc. cit., vol. 2, p. 264.

$\dagger$ Klein-Fricke, loc. cit., vol. 2, pp. 292-93. 
elliptic parameter $u=0, Y_{i}=0$. Therefore, the line $S_{1}(Z)$ meets each $E^{5}$ in the point $u=0$. For the parameters $u=\omega_{1} / 2, \omega_{2} / 2,\left(\omega_{1}+\omega_{2}\right) / 2, Z_{i}=0$. That is, $S_{2}(Y)$ cuts each $E^{5}$ in the three half-period points. Then by planes on $S_{1}(Z)$ the $E^{5}$ 's are projected into doubly covered conics $R^{2}(Y)$ in $S_{2}(Y)$. $^{*}$

Let $t_{1}=Z_{1}(0)$ and $t_{2}=Z_{2}(0)$ for a particular $E^{\mathfrak{b}}$ of the family. Then for $u=0, Z_{i}=t_{i}$ and $Y_{i}=0$. The coefficients of $\zeta$ and $\theta$ in $F_{1}$ and $F_{3}$ vanish identically. Solving $F_{2}$ for $\alpha$ we obtain

$$
\alpha_{0}: \alpha_{1}: \alpha_{2}=t_{1} t_{2}: t_{2}^{2}:-t_{1}^{2} .
$$

Hence for given $t$, that is, for a given point of $S_{1}(Z)$, the five quadrics (2) are known and $E^{5}$ is completely determined. Since the three quadrics in $F_{1}$ and $F_{2}$ vanish for all values of $\zeta$ they necessarily vanish for $\zeta=\alpha$. But $F_{2} \equiv 0$ for $\zeta=\alpha$. Therefore, $F_{1}$ gives a quadric which does not contain the $Z$ 's and hence must be the conic $R^{2}(Y)$ in $S_{2}(Y)$. For $\zeta=\alpha$ and (6), $F_{1}$ becomes the conic*

(7) $t_{1}^{2} t_{2}^{2} Y_{0}^{2}+t_{2}^{4} Y_{0} Y_{2}+t_{1}^{4} Y_{0} Y_{1}+2 t_{1} t_{2}^{3} Y_{1}^{2}-2 t_{1}^{3} t_{2} Y_{2}^{2}-2 t_{1}^{2} t_{2}^{2} Y_{1} Y_{2}=0$.

The parametric representation of $S_{2}(Y)$ in $Z$ and $t$ is obtained from $F_{3}$ in (3) which contains $Y$ linearly:

$$
\begin{aligned}
& \rho Y_{0}=2 Z_{1}^{2} t_{1} t_{2}^{3}+6 Z_{1} Z_{2} t_{1}^{2} t_{2}^{2}+2 Z_{2}^{2} t_{1}^{3} t_{2}, \\
& \rho Y_{1}=Z_{1}^{2} t_{1}^{4}-Z_{1} Z_{2} t_{2}^{4}-2 Z_{2}^{2} t_{1} t_{2}^{3}, \\
& \rho Y_{2}=-2 Z_{1}^{2} t_{1}^{3} t_{2}-Z_{1} Z_{2} t_{1}^{4}-Z_{2}^{2} t_{2}^{4} .
\end{aligned}
$$

For the point $u=0$, or $t_{1}: t_{2}=Z_{1}: Z_{2}$, we obtain in $S_{2}(Y)$

$$
\rho Y_{0}=10 t_{1}^{3} t_{2}^{3}, \quad \rho Y_{1}=t_{1}{ }^{6}-3 t_{1} t_{2}{ }^{5}, \quad \rho Y_{2}=-3 t_{1}{ }^{5} t_{2}-t_{2}{ }^{6} .
$$

This is a rational sextic which is the projection in $S_{2}(Y)$ of the point $u=0$ on the family of $E^{5}$ 's in $S_{4}$. The conics (7) are the osculant conics of the sextic (8).* If $u=\omega_{1} / 2, \omega_{2} / 2,\left(\omega_{1}+\omega_{2}\right) / 2$, the $Z$ 's are zero and hence the locus in $S_{2}(Y)$ of the half period points of $E^{5}$ is obtained by eliminating $\alpha$ from $F_{1}$ in (3). This gives the sextic $\dagger$

$$
Y_{0}^{4} Y_{1} Y_{2}-Y_{0}^{2} Y_{1}^{2} Y_{2}^{2}-Y_{0}\left(Y_{1}^{5}+Y_{2}^{5}\right)+2 Y_{1}^{3} Y_{2}^{8}=0 .
$$

As a quartic in $t$, (7) has the invariants

$$
\begin{aligned}
& g_{2}=(1 / 12)\left(Y_{0}^{2}+4 Y_{1} Y_{2}\right)^{2}, \\
& g_{3}=(1 / 216)\left[-Y_{0}^{6}+42 Y_{0}^{4} Y_{1} Y_{2}-102 Y_{0}^{2} Y_{1}^{2} Y_{2}^{2}-54 Y_{0}\left(Y_{1}^{6}+Y_{2}^{5}\right)+44 Y_{1}^{8} Y_{2}^{3}\right] .
\end{aligned}
$$

* Miller, loc. cit., p. 279.

$\dagger$ Miller, loc. cit., p. 283. 
The conic

$$
Y_{0}^{2}+4 Y_{1} Y_{2}=0
$$

is then an invariant of $G_{60}$ in $S_{2}(Y)$. Moreover

$$
\begin{aligned}
& 12^{3}\left(g_{2}^{8}-27 g_{3}^{2}\right)=54\left[Y_{0}^{4} Y_{1} Y_{2}-Y_{0}^{2} Y_{1}^{2} Y_{2}^{2}+2 Y_{1}^{3} Y_{2}^{3}-Y_{0}\left(Y_{1}^{5}+Y_{2}^{5}\right)\right] \\
& \quad \cdot\left\{-2\left[-Y_{0}^{6}+15 Y_{0}^{4} Y_{1} Y_{2}-75 Y_{0}^{2} Y_{1}^{2} Y_{2}^{2}-10 Y_{1}^{3} Y_{2}^{3}-27 Y_{0}\left(Y_{1}^{5}+Y_{2}^{5}\right)\right]\right\} .
\end{aligned}
$$

This discriminant gives the envelope of the conics (7). Since the first factor is obviously the locus of the half-period points (9), and since the osculant conics touch the sextic (8), the second factor must be the point equation of the rational sextic (8). These sextics must be invariants of $G_{60}$ in $S_{2}(Y)$.

The quadrics on $E^{5}$ in $S_{4}$ can be expressed in terms of identical covariants and the $(3,3)$ form $(1)$, where $t_{1}: t_{2}$ of $(1)$ is replaced by $t_{1}:-t_{2}$ and $\tau_{1}: \tau_{2}$ of (1) is replaced by $\theta_{2}: \theta_{1}$. With this change of variables (1) assumes the form

$$
G=\theta_{1} \theta_{2}^{2} t_{1}^{3}-\theta_{1}^{3} t_{1}^{2} t_{2}+\theta_{2}^{3} t_{1} t_{2}^{2}+\theta_{1}^{2} \theta_{2} t_{2}^{3} .
$$

Put the conic (10) in parametric form

$$
Y_{0}: Y_{1}: Y_{2}=2 \theta_{1} \theta_{2}:-\theta_{1}^{2}: \theta_{2}^{2} \text {. }
$$

Then

$$
F_{3}=3\left\{\theta_{1} \theta_{2}^{2} t_{1}^{3}-\theta_{1}^{3} t_{1}^{2} t_{2}+\theta_{2}^{3} t_{1} t_{2}^{2}+\theta_{1}^{2} \theta_{2} t_{2}^{3}\right\}_{\theta^{\prime} . z^{\prime}}
$$

where \{\}$_{\theta^{\prime}, Z^{\prime}}$ indicates polarization once with respect to $\theta$ and once with respect to $Z$. After the polarization, the $\theta$ 's and $t$ 's must be replaced by $Y^{\prime}$ 's and $\alpha$ 's from (12) and (6) respectively.

$$
F_{2}=(Z t)\left(Z t^{\prime}\right)\left(t t^{\prime}\right)
$$

if $\alpha$ and $\zeta$ are expressed in terms of $t$ and $t^{\prime}$ respectively from (6). If $G$ is written as $(a \theta)^{3}(\alpha t)^{3}$, it has the covariant*

$$
\begin{aligned}
H & =\left(a a^{\prime}\right)(a \theta)^{2}\left(a^{\prime} \theta\right)^{2}\left(\alpha \alpha^{\prime}\right)(\alpha t)^{2}\left(\alpha^{\prime} t\right)^{2} \\
& =t_{1}^{4} \theta_{1}^{3} \theta_{2}+t_{1}^{3} t_{2} \theta_{2}^{4}-3 t_{1}^{2} t_{2}^{2} \theta_{1}^{2} \theta_{2}^{2}-t_{1} t_{2}^{3} \theta_{1}^{4}-t_{2}^{4} \theta_{1} \theta_{2}^{3} .
\end{aligned}
$$

Then

$$
F_{1}\left(Y, Y^{\prime}\right)=-2 H\left(\theta^{2} \theta^{\prime 2}, t^{2} t^{\prime 2}\right)+\frac{1}{3}\left(t t^{\prime}\right)^{2}\left(\theta \theta^{\prime}\right)^{2},
$$

where $F_{1}\left(Y, Y^{\prime}\right)$ indicates polarization once with respect to $Y$ and $H\left(\theta^{2} \theta^{\prime 2}\right.$, $\left.t^{2} t^{\prime 2}\right)$ indicates polarization twice with respect to $\theta$ and twice with respect to $t$. Hence the family of $E^{5}$ 's with a common $G_{50}$ can be expressed algebraically in terms of the $(3,3)$ form $F$ and its covariants, and identical covariants.

* Klein, Vorlesungen über das Ikosaeder, p. 196. 


\section{The $(3,3)$ FORM AND two cubic curves IN SPACE}

We now interpret the $(3,3)$ form $F=0$ as the incidence condition of point $\tau$ of the space cubic curve $c_{1}(\tau)$ and plane $t$ of the space cubic curve $c_{2}(t)$. Let the coördinate system be chosen so that $c_{1}(\tau)$ has the equations

$$
x_{0}=\tau^{3}, x_{1}=3 \tau^{2}, \quad x_{2}=3 \tau, x_{3}=1 \text {, }
$$

and

$$
\xi_{0}=1, \quad \xi_{1}=-\tau, \quad \xi_{2}=\tau^{2}, \quad \xi_{3}=-\tau^{3},
$$

in points and planes respectively. If $F=0$ is the incidence condition of point $\tau$ of $c_{1}(\tau)$ and plane $t$ of $c_{2}(t)$, then

$$
\xi_{0}=t, \quad \xi_{1}=\frac{1}{3} t^{3}, \quad \xi_{2}=-\frac{1}{3}, \quad \xi_{3}=t^{2},
$$

and

$$
x_{0}=t^{2}, \quad x_{1}=1, \quad x_{2}=t^{3}, \quad x_{3}=-t,
$$

are the equations of $c_{2}(t)$ in planes and points respectively. However, the incidence condition of point $t$ of $c_{2}(t)$ and plane $\tau$ of $c_{1}(\tau)$ becomes $\bar{F}=t^{2}-\tau$ $+t^{3} \tau^{2}+t \tau^{3}=0$. But $\bar{F} \equiv F$. Hence the point $\tau$ of $c_{1}(\tau)$ lies on plane $t$ of $c_{2}(t)$ and point $t$ of $c_{2}(t)$ lies on plane $\tau$ of $c_{1}(\tau)$ if $F=0$.

The two cubic curves have this further property in common. They both lie in the same null system whose equations are

$$
\rho u_{0}=3 x_{3}, \quad \rho u_{1}=-x_{2}, \quad \rho u_{2}=x_{1}, \quad \rho u_{3}=-3 x_{0} .
$$

For convenience we here identify part of the covariants given by Coble* for the general case with those given by Gordantf:

$$
\begin{aligned}
& c_{4,4}^{(2)}=(4 / 9)\left[t_{1}^{4} \tau_{1} \tau_{2}^{8}-t_{1}^{3} t_{2} \tau_{1}^{4}-3 t_{1}^{2} t_{2}^{2} \tau_{1}^{2} \tau_{2}^{2}+t_{1} t_{2}^{8} \tau_{2}^{4}-t_{2}^{4} \tau_{1} \tau_{2}\right], \\
& c_{2,6}^{(2)}=-(2 / 9)\left[t_{1}{ }^{6} \tau_{1}^{2}-3 t_{1} t_{2} \tau_{2}^{2}-10 t_{1}{ }^{3} t_{2}^{3} \tau_{1} \tau_{2}+3 t_{1} t_{2}{ }^{5} \tau_{1}^{2}+t_{2}{ }^{6} \tau_{2}^{2}\right], \\
& c_{6,2}^{(2)}=-(2 / 9)\left[t_{1}^{2}\left(\tau_{2}^{6}-3 \tau_{1}^{5} \tau_{2}\right)+10 t_{1} t_{2} \tau_{1}^{3} \tau_{2}^{8}+t_{2}^{2}\left(\tau_{1}{ }^{6}+3 \tau_{1} \tau_{2}^{5}\right)\right], \\
& c_{0,4}^{(2)} \equiv 0, \quad c_{4,0}^{(2)} \equiv 0, \quad c_{0,0}^{(2)}=4 / 3 .
\end{aligned}
$$

Since there is a group of order 120 for which $F$ is invariant, the space con-

* American Journal of Mathematics, vol. 46, pp. 147-48.

$\dagger$ Mathematische Annalen, vol. 13, pp. 386-87.

$\ddagger$ By using these values to check the syzygies connecting the comitants of the second degree given by Coble (American Journal of Mathematics, vol. 46, p. 155, (18)) it is found that the last one is wrong. It should read

$$
24\left(c c^{\prime}\right)^{4} \equiv 24\left(\gamma \gamma^{\prime}\right)^{4} \equiv 180 \delta^{2}-\left(b b^{\prime}\right)^{4}\left(\beta \beta^{\prime}\right)^{4} .
$$

The theorem should read "The forty coefficients of these syzygies furnish the forty linearly independent relations of the second degree among the two-row minors of $R$, etc. 
figuration must be invariant under a group $G_{120}$. In the group on $F$ the largest subgroup which transforms $t$ into $t^{\prime}$ and $\tau$ into $\tau^{\prime}$ is the icosahedral $G_{60}$. The further operations interchange $t$ and $\tau$. Hence in the space configuration each space cubic curve is unaltered by an icosahedral $G_{60}$. The operations which interchange $t$ and $\tau$ will then interchange the two cubic curves.

The $G_{60}$ is generated by $S$ and $T^{*}$ :

$$
S: \begin{array}{ll}
t_{1}^{\prime}=\epsilon t_{1}, & \tau_{1}^{\prime}=\epsilon^{2} \tau_{1} \\
t_{2}^{\prime}=\epsilon^{4} t_{2}, & \tau_{2}^{\prime}=\epsilon^{3} \tau_{2}
\end{array}
$$

$T: \begin{array}{ll}5^{1 / 2} t_{1}^{\prime}=-\left(\epsilon^{2}-\epsilon^{3}\right) t_{1}+\left(\epsilon^{4}-\epsilon\right) t_{2}, & 5^{1 / 2} \tau_{1}^{\prime}=\left(\epsilon^{4}-\epsilon\right) \tau_{1}+\left(\epsilon^{2}-\epsilon^{3}\right) \tau_{2}, \\ 5^{1 / 2} t_{2}^{\prime}=\left(\epsilon^{4}-\epsilon\right) t_{1}+\left(\epsilon^{2}-\epsilon^{3}\right) t_{2}, & 5^{1 / 2} \tau_{2}^{\prime}=\left(\epsilon^{2}-\epsilon^{3}\right) \tau_{1}-\left(\epsilon^{4}-\epsilon\right) \tau_{2} ;\end{array}$

and, for convenience,

$$
U: \begin{array}{ll}
t_{1}^{\prime}=-t_{2}, & \tau_{1}^{\prime}=-\tau_{2}, \\
t_{2}^{\prime}=t_{1}, & \tau_{2}^{\prime}=\tau_{1} .
\end{array}
$$

Then as a collineation group on the $x$ 's the operations of $G_{60}$ are

$$
S^{\mu}: \begin{array}{ll}
x_{0}^{\prime}=\epsilon^{\mu} x_{0}, & x_{0}^{\prime}=-\epsilon^{4 \mu} x_{3}, \\
x_{1}^{\prime}=\epsilon^{2 \mu} x_{1}, & S^{\mu} U: \begin{array}{l}
x_{1}^{\prime}=\epsilon^{3 \mu} x_{2}, \\
x_{2}^{\prime}=\epsilon^{3 \mu} x_{2},
\end{array} \\
x_{2}^{\prime}=\epsilon^{4 \mu} x_{3} ; & x_{3}^{\prime}=\epsilon^{\mu} x_{0} ;
\end{array}
$$

$S^{\mu} T S^{\nu}: \quad 5 \cdot 5^{1 / 2} x_{0}^{\prime}=\epsilon^{\prime}\left[-\left(\epsilon-\epsilon^{4}\right)^{3} \epsilon^{\mu} x_{0}-5^{1 / 2}\left(\epsilon-\epsilon^{4}\right) \epsilon^{2 \mu} x_{1}+5^{1 / 2}\left(\epsilon^{2}-\epsilon^{3}\right) \epsilon^{3 \mu} x_{2}\right.$

$$
\begin{gathered}
\left.+\left(\epsilon^{2}-\epsilon^{3}\right)^{3} \epsilon^{4 \mu} x_{3}\right], \\
5 \cdot 5^{1 / 2} x_{1}^{\prime}=\epsilon^{2 \nu}\left[-3 \cdot 5^{1 / 2}\left(\epsilon-\epsilon^{4} \epsilon^{\mu} x_{0}+\left(\epsilon^{2}-\epsilon^{3}\right)^{3} \epsilon^{2 \mu} x_{1}-\left(\epsilon-\epsilon^{4}\right)^{3} \epsilon^{3 \mu} x_{2}\right.\right.
\end{gathered}
$$$$
\left.-3 \cdot 5^{1 / 2}\left(\epsilon^{2}-\epsilon^{3}\right) \epsilon^{4 \mu} x_{3}\right] \text {, }
$$$$
5 \cdot 5^{1 / 2} x_{2}^{\prime}=\epsilon^{3 \nu}\left[3 \cdot 5^{1 / 2}\left(\epsilon^{2}-\epsilon^{3}\right) \epsilon^{\mu} x_{0}-\left(\epsilon-\epsilon^{4}\right)^{3} \epsilon^{2 \mu} x_{1}-\left(\epsilon^{2}-\epsilon^{3}\right)^{3} \epsilon^{3 \mu} x_{2}\right.
$$$$
\left.-3 \cdot 5^{1 / 2}\left(\epsilon-\epsilon^{4}\right) \varepsilon^{4 \mu} x_{3}\right] \text {, }
$$$$
5 \cdot 5^{1 / 2} x_{3}^{\prime}=\epsilon^{4 \nu} \cdot\left[\left(\epsilon^{2}-\epsilon^{3}\right)^{3} \epsilon^{\mu} x_{0}-5^{1 / 2}\left(\epsilon^{2}-\epsilon^{3}\right) \epsilon^{2 \mu} x_{1}-5^{1 / 2}\left(\epsilon-\epsilon^{4}\right) \epsilon^{3 \mu} x_{2}\right.
$$$$
\left.+\left(\epsilon-\epsilon^{4}\right)^{3} \epsilon^{4 \mu} x_{3}\right]
$$

$$
\begin{aligned}
& S^{\mu} T S^{\nu} U: 5 \cdot 5^{1 / 2} x_{0}^{\prime}=-\epsilon^{4 \nu}\left[\left(\epsilon^{2}-\epsilon^{3}\right)^{3} \epsilon^{\mu} x_{0}-5^{1 / 2}\left(\epsilon^{2}-\epsilon^{3}\right) \epsilon^{2 \mu} x_{1}-5^{1 / 2}\left(\epsilon-\epsilon^{4}\right) \epsilon^{3 \mu} x_{2}\right. \\
& \left.+\left(\epsilon-\epsilon^{4}\right)^{3} \epsilon^{4 \mu} x_{3}\right] \text {, } \\
& 5 \cdot 5^{1 / 2} x_{1}^{\prime}=\epsilon^{30}\left[3 \cdot 5^{1 / 2}\left(\epsilon^{2}-\epsilon^{3}\right) \epsilon^{\mu} x_{0}-\left(\epsilon-\epsilon^{4}\right)^{3} \epsilon^{2 \mu} x_{1}-\left(\epsilon^{2}-\epsilon^{3}\right)^{3} \epsilon^{3 \mu} x_{2}\right. \\
& \left.-3 \cdot 5^{1 / 2}\left(\epsilon-\epsilon^{4}\right) \epsilon^{4 \mu} x_{3}\right] \text {, }
\end{aligned}
$$

\footnotetext{
* Gordan, Mathematische Annalen, loc. cit., p. 379.
} 


$$
\begin{aligned}
5 \cdot 5^{1 / 2} x_{2}^{\prime}= & -\epsilon^{2 \nu}\left[-3 \cdot 5^{1 / 2}\left(\epsilon-\epsilon^{4}\right) \epsilon^{\mu} x_{0}+\left(\epsilon^{2}-\epsilon^{3}\right)^{3} \epsilon^{2 \mu} x_{1}-\left(\epsilon-\epsilon^{4}\right)^{3} \epsilon^{3 \mu} x_{2}\right. \\
& \left.-3 \cdot 5^{1 / 2}\left(\epsilon^{2}-\epsilon^{3}\right) \epsilon^{4 \mu} x_{3}\right], \\
5 \cdot 5^{1 / 2} x_{3}^{\prime}= & \epsilon^{\nu}\left[-\left(\epsilon-\epsilon^{4}\right)^{3} \epsilon^{\mu} x_{0}-5^{1 / 2}\left(\epsilon-\epsilon^{4}\right) \epsilon^{2 \mu} x_{1}+5^{1 / 2}\left(\epsilon^{2}-\epsilon^{3}\right) \epsilon^{3 \mu} x_{2}\right. \\
& \left.+\left(\epsilon^{2}-\epsilon^{3}\right)^{3} \epsilon^{4 \mu} x_{3}\right] \quad(\mu, \nu=0,1,2,3,4) .
\end{aligned}
$$

The operation which interchanges $t$ and $\tau$ and leaves $F$ invariant is

$$
R: t_{1}^{\prime}=\tau_{1}, \quad t_{2}^{\prime}=\tau_{2}, \quad \tau_{1}^{\prime}=-t_{2}, \quad \tau_{2}^{\prime}=t_{1},
$$

where $R^{2}=U$. As a collineation in space for which a point of one cubic curve goes into a point of the other, $R$ becomes

$$
3^{1 / 2} x_{0}^{\prime}=-x_{1}, 3^{1 / 2} x_{1}^{\prime}=-3 x_{3}, 3^{1 / 2} x_{2}^{\prime}=-3 x_{0}, 3^{1 / 2} x_{3}^{\prime}=x_{2} .
$$

The quaternary icosahedral $G_{60}$ 's and the symmetric $G_{120}$ 's are listed in the literature but it is not shown that an icosahedral $G_{60}$ leaves each of two space cubic curves unaltered and a symmetric $G_{120}$ interchanges these two curves.

This collineation group may be enlarged to a correlation group by using $R$ to send point and plane of $c_{1}(\tau)$ into plane and point of $c_{2}(t)$ respectively and vice versa. The correlation is, then,

$$
C: \begin{aligned}
& \xi_{0}^{\prime}=x_{2}, \quad \xi_{1}^{\prime}=x_{0}, \quad \xi_{2}^{\prime}=-x_{3}, \quad \xi_{3}^{\prime}=x_{1}, \\
& x_{0}^{\prime}=\xi_{2}, \quad x_{1}^{\prime}=\xi_{0}, \quad x_{2}^{\prime}=-\xi_{3}, \quad x_{3}^{\prime}=\xi_{1} .
\end{aligned}
$$

III. The Rational ICOSAhedRal SEXTIC

The nets of point quadrics on $c_{1}(\tau)$ and $c_{2}(t)$ are, respectively,

$$
Q_{1}: u_{0}\left(x_{1}^{2}-3 x_{0} x_{2}\right)+u_{1}\left(x_{1} x_{2}-9 x_{0} x_{3}\right)+u_{2}\left(x_{2}^{2}-3 x_{1} x_{3}\right)=0,
$$

and

$$
Q_{2}: u_{0}\left(x_{0}^{2}+x_{2} x_{3}\right)+u_{1}\left(-x_{1} x_{2}-x_{0} x_{3}\right)+u_{2}\left(x_{3}^{2}-x_{0} x_{1}\right)=0,
$$

and the nets of quadric envelopes on the planes of $c_{1}(\tau)$ and $c_{2}(t)$ are, respectively,

and

$$
\bar{Q}_{1}: \quad u_{0}\left(\xi_{2}^{2}-\xi_{1} \xi_{3}\right)+u_{1}\left(\xi_{0} \xi_{3}-\xi_{1} \xi_{2}\right)+u_{2}\left(\xi_{1}^{2}-\xi_{0} \xi_{2}\right)=0
$$

$$
\bar{Q}_{2}: u_{0}\left(\xi_{3}^{2}-3 \xi_{0} \xi_{1}\right)+u_{1}\left(\xi_{0} \xi_{3}+9 \xi_{1} \xi_{2}\right)+u_{2}\left(\xi_{0}^{2}+3 \xi_{2} \xi_{3}\right)=0 .
$$

The net $Q_{1}$ will cut $c_{2}(t)$ in a linear series $I_{2}^{6}$ determined by substituting the coördinates of $c_{2}(t)$ in $Q_{1}{ }^{*}$ This gives

$$
u_{0}\left(1-3 t^{5}\right)+u_{1}\left(10 t^{3}\right)+u_{2}\left(t^{6}+3 t\right)=0 .
$$

* Coble, American Journal of Mathematics, vol. 46, p. 156. 
Then (1) may be interpreted as the line sections of a rational plane sextic $S_{2}(t)$ whose point equations are

$$
x_{0}=1-3 t^{5}, \quad x_{1}=10 t^{3}, \quad x_{2}=t^{6}+3 t .
$$

$\bar{Q}_{1}$ on $c_{2}(t)$ is found to give the same sextic, that is, $\bar{S}_{2}(t) \equiv S_{2}(t){ }^{*}$ The linear series cut out on $c_{1}(\tau)$ by $Q_{2}$ and $\bar{Q}_{2}$ give the sextics $\bar{S}_{1}(\tau) \equiv S_{1}(\tau)$ :

$$
x_{0}=\tau^{6}+3 \tau, \quad x_{1}=-10 \tau^{3}, \quad x_{2}=1-3 \tau^{5} .
$$

That is, instead of the four sextics of the general case* there are only two for the special case. In the same plane these two sextics are also identical since both point equations are $\dagger$

$$
27 x_{1}\left(x_{0}{ }^{5}-x_{2}^{5}\right)-10 x_{0}^{3} x_{2}^{3}+75 x_{0}^{2} x_{1}^{2} x_{2}^{2}+15 x_{0} x_{1}^{4} x_{2}+x_{1}^{6}=0 .
$$

Corresponding to the ten nodes of the sextics are the ten common bisecants and the ten common axes of the curves $c_{1}(\tau)$ and $c_{2}(t)$ since the two points which come together at a node of the sextic do not determine a line section of the sextic but a pencil of line sections.

The cones of the net $Q_{1}$ are given when the constants satisfy the condition

$$
u_{0} u_{2}-u_{1}^{2}=0 \text {. }
$$

That is, to the cones in $Q_{1}$ correspond the lines of a conic in the plane of the sextic. The equations of the cones become

$$
u_{1}^{2}\left(x_{1}^{2}-3 x_{0} x_{2}\right)+u_{1} u_{2}\left(x_{1} x_{2}-9 x_{0} x_{3}\right)+u_{2}^{2}\left(x_{2}^{2}-3 x_{1} x_{3}\right)=0 .
$$

The corresponding lines in the plane satisfy (3) and

$$
u_{1}^{2}\left(1-3 t^{5}\right)+u_{1} u_{2}\left(10 t^{3}\right)+u_{2}^{2}\left(t^{6}+3 t\right)=0 .
$$

In the plane of $S_{2}(t)$ there is a conic such that for every point of the sextic there are two lines of the conic and for every line of the conic there are six points of the sextic. If the equations of the conic (3) are

$$
K(\tau): \begin{array}{lll}
x_{0}=\tau^{2}, & x_{1}=2 \tau, & x_{2}=1, \\
u_{0}=1, & u_{1}=-\tau, & u_{2}=\tau^{2},
\end{array}
$$

in points and lines respectively, this $(2,6)$ relation is obtained from (1) as

$$
\left(1-3 t^{5}\right)-\tau\left(10 t^{3}\right)+\tau^{2}\left(t^{6}+3 t\right)=0
$$

which is evidently $c_{2,6}^{(2)}=0$, of (3) $\S I I$. (4) is then the equation of the sextic $S_{2}(t) \equiv \bar{S}_{2}(t)$ in Darboux coördinates; that is,

* Coble, American Journal of Mathematics, vol. 46, p. 157.

† Winger, Mathematische Annalen, loc. cit., p. 211. 


$$
x_{0}=\tau_{1} \tau_{2}, x_{1}=\tau_{1}+\tau_{2}, x_{2}=1 .
$$

To a pencil of lines in the plane on a point of $S_{2}(t)$ corresponds a pencil of quadrics in $Q_{1}$ on a bisecant of $c_{1}(\tau)$ on a point of $c_{2}(t)$. To the tangents of the conic in the pencil of lines correspond the cones in the pencil of quadrics. If a point is a node of $S_{2}(t)$ the pencil of quadrics is the pencil on a common bisecant of $c_{1}(\tau)$ and $c_{2}(t)$ and the cones have vertices at the points of intersection of this common bisecant with $c_{1}(\tau)$, say at $\tau_{1}$ and $\tau_{2}$. Then $\tau_{1}$ and $\tau_{2}$ give two tangents on $K(t)$ which intersect in a node of the sextic $S_{1}(\tau)$.*

The sextic $S_{2}(t)$ is evidently the sextic (8) $\$ I$, except for a change in notation, which is invariant under the ternary icosahedral group. The ten double points are the ten points isomorphic with the ten diagonals of the dodecahedron. The coördinates of the points in the notation used here are

$$
\left(-\epsilon^{4 i}, \delta_{j}{ }^{2}, \epsilon^{i}\right) \quad(i=0,1,2,3,4 ; j=1,2)
$$

where

$$
\delta_{1}=\epsilon+\epsilon^{4}, \delta_{2}=\epsilon^{2}+\epsilon^{3} .
$$

Winger notes the fact that the double points lie by sixes on a set of ten conics associated with the dihedral $G_{6}$ 's in the $G_{60} . \dagger$ However, they lie by sixes on another set of fifteen conics associated with the fifteen reflexions in the $G_{60}$.

Name the diagonals of the icosahedron 1, 2, 3, 4, 5, 6. Then the double points of the sextic which correspond to the diagonals of the dodecahedron may be named by the three adjacent icosahedral diagonals, that is, $(i j k)$. Let the diagonals of the icosahedron be named in such a manner that the double points will be

$$
\begin{array}{ll}
\text { 1, (123), }\left(-\epsilon^{3}, \delta_{2}{ }^{2}, \epsilon^{2}\right), & \text { 6, (245), }\left(-1, \delta_{1}{ }^{2}, 1\right), \\
\text { 2, (134), }\left(-\epsilon^{4}, \delta_{2}{ }^{2}, \epsilon\right), & \text { 7, (356), }\left(-\epsilon, \delta_{1}{ }^{2}, \epsilon^{4}\right), \\
\text { 3, (145), }\left(-1, \delta_{2}{ }^{2}, 1\right), & \text { 8, (246), }\left(-\epsilon^{2}, \delta_{1}{ }^{2}, \epsilon^{3}\right), \\
\text { 4, (156), }\left(-\epsilon, \delta_{2}{ }^{2}, \epsilon^{4}\right), & \text { 9, (235), }\left(-\epsilon^{3}, \delta_{1}{ }^{2}, \epsilon^{2}\right), \\
\text { 5, (162), }\left(-\epsilon^{2}, \delta_{2}{ }^{2}, \epsilon^{3}\right), & \text { 0, (346), }\left(-\epsilon^{4}, \delta_{1}{ }^{2}, \epsilon\right) .
\end{array}
$$

The second column which gives the double points with reference to the icosahedral diagonals also gives an interesting number system. It is a triad system with double couples unaltered by a $G_{60}$ given by Emch. $\neq$

The icosahedral group may be written as a substitution group on the six diagonals generated by $S=(23456)$ and $T=(12)(36)$. Under the operations

* Coble, American Journal of Mathematics, vol. 46, p. 157.

$\dagger$ American Journal of Mathematics, loc. cit., p. 56.

$\ddagger$ A. Emch, Triple and multiple systems, their geometric configurations and groups, these Transactions, vol. 31 (1929), pp. 25-42. 
of period two, two of the double points are fixed and the remaining eight are separated into four pairs. If a conic passes through one of each of three pairs and the mates of two of the three, it must pass through the mate of the third from the symmetry of the configuration. In this way we find two sets of conjugate conics, one containing ten and the other fifteen, which pass through six double points.

The conics in the set of ten are the following:

$\begin{array}{ll}(123568), & (126890), \\ (134569), & (456789), \\ (124570), & (236790), \\ (234580), & (157890), \\ (123479), & (346780) .\end{array}$

These conics may be associated with the ten diagonals of the dodecahedron. Three edges of the dodecahedron meet at the vertex $(i j k)$. The three adjacent vertices are named $(i j l),(i k m),(j k n)$. These four vertices represent a set of four nodes. The remaining six lie on a conic.

The conics forming the set of fifteen are the following:

$\begin{array}{lll}(124589), & (234567), & (256780), \\ (234689), & (123460), & (356890), \\ (123780), & (145680), & (136789), \\ (134578), & (123590), & (146790), \\ (125679), & (345790), & (247890) .\end{array}$

These conics may be associated with the fifteen cross lines of the dodecahedron or with the fifteen reflexions in the $G_{60}$. The four vertices of the dodecahedron, consisting of the two on any edge and the vertices opposite this edge in each of the adjoining faces, represent a set of four nodes. From the manner in which the figure is numbered, the opposite edge gives the same set of four nodes. The remaining six nodes lie on a conic.

The numbers which give these conics also form number systems. The set of ten conics gives the arrangement of ten numbers six at a time such that each number occurs in six. The set of fifteen conics gives a number system with double couples. It gives the arrangement of ten numbers six at a time such that each number occurs nine times and each couple occurs five times. These number systems are unaltered by a group of order 120 .

IV. The CUBIC ENVELopes PeRspective to the Rational SEXtiC

The planes of $c_{1}(\tau)$ cut any plane, $\tau_{0}$, of $c_{1}(\tau)$ in a line conic which may be taken to be the conic

$$
K(\tau): \quad u_{0} u_{2}-u_{1}^{2}=0 \text { or } 4 x_{0} x_{2}-x_{1}^{2}=0 .
$$


This conic may be used as a coördinate system in the plane $\tau_{0}$. Any two planes, $\tau_{1}$ and $\tau_{2}$, of $c_{1}(\tau)$ intersect in an axis of $c_{1}(\tau)$. These two planes cut $\tau_{0}$ in two lines of the conic (1). These two lines intersect in the point $x$ where the axis cuts the plane $\tau_{0}$. The coördinates of this point $x$ will be

$$
x_{0}=\tau_{1} \tau_{2}, x_{1}=\tau_{1}+\tau_{2}, x_{2}=1 .
$$

The planes $t$ of $c_{2}(t)$ cut the planes of $c_{1}(\tau)$ in cubic envelopes which may be projected by the axes of $c_{1}(\tau)$ onto a plane $\tau_{0}$ of $c_{1}(\tau)$ to give a pencil of cubic envelopes in $\tau_{0}{ }^{*}$ If $c_{1}(\tau)$ be chosen as a reference system the plane $t$ of $c_{2}(t)$ is the plane

$$
F=(\alpha t)^{3}(a \tau)^{3}=t_{1}^{3} \tau_{1}^{2} \tau_{2}+t_{1}^{2} t_{2} \tau_{2}^{3}+t_{1} t_{2}^{2} \tau_{1}^{3}-t_{2}^{3} \tau_{1} \tau_{2}^{2}=0 .
$$

The point $\tau_{1}, \tau_{2}, \tau$ is on this plane if

$$
\left(a \tau_{1}\right)\left(a \tau_{2}\right)(a \tau)(\alpha t)^{3}=0 .
$$

Polarizing $F$ in (3) with respect to $\tau_{1}$ and $\tau_{2}$, we obtain

$$
\tau_{1} \tau_{2}\left(t^{3}+3 t \tau\right)+\left(\tau_{1}+\tau_{2}\right)\left(t^{3} \tau-1\right)+\left(3 t^{2}-\tau\right)=0 .
$$

Substituting (2), we obtain

$$
x_{0}\left(t^{3}+3 t \tau\right)+x_{1}\left(t^{3} \tau-1\right)+x_{2}\left(3 t^{2}-\tau\right)=0 .
$$

That is, the pencil of cubic envelopes cut out by the planes of $c_{2}(t)$ on a plane of $c_{1}(\tau)$ are

$$
E_{3}(t): \quad \xi_{0}=t^{3}+3 t \tau, \quad \xi_{1}=t^{3} \tau-1, \quad \xi_{2}=3 t^{2}-\tau .
$$

For $\tau=\tau_{1}$ and $\tau_{2}$ in (4), we have the lines

$$
\begin{aligned}
& x_{0}\left(t^{3}+3 t \tau_{1}\right)+x_{1}\left(t^{3} \tau_{1}-1\right)+x_{2}\left(3 t^{2}-\tau_{1}\right)=0, \\
& x_{0}\left(t^{3}+3 t \tau_{2}\right)+x_{1}\left(t^{3} \tau_{2}-1\right)+x_{2}\left(3 t^{2}-\tau_{2}\right)=0,
\end{aligned}
$$

respectively, of the cubic envelopes (5). These lines intersect in the point

$$
x_{0}: x_{1}: x_{2}=1-3 t^{5}: 10 t^{3}: 3 t+t^{6} .
$$

Since the coördinates of this point are independent of $\tau$, for given $t$ all the lines of the $\infty^{1}$ cubic envelopes pass through the same point (6). For variable $t$ this point describes the rational sextic (2) §III.

In every plane $t$ of $c_{2}(t)$ there is an axis of $c_{1}(\tau)$. Since $F=0$ gives the points where plane $t$ cuts $c_{1}(\tau)$ the Hessian of $F$ as a cubic in $\tau$ gives the parameters of the planes which intersect in an axis in plane $t$. The Hessian of $F$ is from (3) §II

\footnotetext{
* Coble, American Journal of Mathematics, vol. 46, p. 160.
} 


$$
\tau_{1}^{2}\left(t_{1}^{6}+3 t_{1} t_{2}^{5}\right)-10 t_{1}^{3} t_{2}^{8} \tau_{1} \tau_{2}+\tau_{2}^{2}\left(t_{2}{ }^{6}-3 t_{1}{ }^{5} t_{2}\right)=0 .
$$

But this is also the rational sextic (2) §III in Darboux coördinates. That is, the rational sextic (2) §III is the locus of points where axes of $c_{1}(\tau)$ in planes of $c_{2}(t)$ cut the plane $\tau_{0}$ and is perspective to the cubic envelopes in $\tau_{0}$ cut out by the planes of $c_{2}(t){ }^{*}$

The incidence condition of point $t$ of $S_{2}(t)$ and line $t^{\prime}$ of $E_{3}(t)$ becomes (in homogeneous parameters)

$$
\begin{gathered}
\left(t_{1} t_{2}^{\prime}-t_{2} t_{1}^{\prime}\right)\left[\tau_{1}\left(-t_{1}^{5} t_{2}^{\prime 2}-10 t_{1}^{4} t_{2} t_{1}^{\prime} t_{2}^{\prime}-10 t_{1}^{3} t_{2}^{2} t_{1}^{\prime 2}-3 t_{2}^{5} t_{2}^{\prime 2}\right)\right. \\
\left.+\tau_{2}\left(3 t_{1}^{5} t_{1}^{\prime 2}-10 t_{1}^{2} t_{2}^{8} t_{2}^{\prime 2}-10 t_{1} t_{2}^{4} t_{1}^{\prime} t_{2}^{\prime}-t_{2}{ }^{5} t_{1}^{\prime 2}\right)\right]=0 .
\end{gathered}
$$

For a node of $S_{2}(t)$ the incidence condition must contain a quadratic in $t$. For $\left(-\epsilon^{4 i}, \delta_{7}^{2}, \epsilon^{i}\right)$, the condition is

(8) $\left(-\epsilon^{3 i} t_{1}^{2}+\delta_{k}^{2} t_{1} t_{2}+\epsilon^{2 i} t_{2}^{2}\right)\left[\delta_{k}^{2}\left(\epsilon^{4} t_{2} \tau_{1}-\epsilon^{i} t_{1} \tau_{2}\right)+\left(\epsilon^{2 i} t_{1} \tau_{1}+\epsilon^{3 i} t_{2} \tau_{2}\right)\right]=0, j \neq k$.

The quadratic factors in $t$ in (8) equated to zero give the nodal parameters of $S_{2}(t)$. If the two roots of one of these quadratic equations are located on the conic

$$
K(t): \quad x_{0}=t^{2}, x_{1}=2 t, x_{2}=1,
$$

the tangents at these points intersect in the point $x_{0}: x_{1}: x_{2}=t_{1} t_{2}: t_{1}+t_{2}: 1$. The ten quadratics in (8) give the points $\left(-\epsilon^{2 i}, \delta_{k}^{2}, \epsilon^{3 i}\right)$, which are the nodes of $S_{1}(\tau)$.

Lines on two nodes cut the sextic in two more points whose parameters are given by quadratics in $t$ obtained by eliminating $\tau$ from the bilinear forms in (8). Locating these with respect to $K(t)$ as above we have the points

$$
\begin{array}{ll}
\left(\epsilon^{i}, 0,1\right), & {\left[-\epsilon^{i}\left(\delta_{2}+\epsilon^{2}\right), \delta_{1}, \epsilon^{4 i}\left(\delta_{2}+\epsilon^{3}\right)\right],} \\
\left(-\epsilon^{i}, \delta_{i}-3, \epsilon^{4 i}\right), & {\left[-\epsilon^{i}\left(\delta_{2}+\epsilon^{3}\right), \delta_{1}, \epsilon^{4 i}\left(\delta_{2}+\epsilon^{2}\right)\right],} \\
\left(-\epsilon^{i}, 2 \delta_{j}, \epsilon^{4 i}\right), & {\left[-\epsilon^{i}\left(\delta_{1}+\epsilon\right), \delta_{2}, \epsilon^{4 i}\left(\delta_{1}+\epsilon^{4}\right)\right],} \\
& {\left[-\epsilon^{i}\left(\delta_{1}+\epsilon^{4}\right), \delta_{2}, \epsilon^{4 i}\left(\delta_{1}+\epsilon\right)\right]} \\
& (i=0,1,2,3,4 ; j=1,2) .
\end{array}
$$

Fifteen of these points, that is,

$$
\left(\epsilon^{i}, 0,1\right), \quad\left(-\epsilon^{i}, 2 \delta_{1}, \epsilon^{4 i}\right), \quad\left(-\epsilon^{i}, 2 \delta_{2}, \epsilon^{4 i}\right)
$$

in the $t$-plane are the fifteen points which form a conjugate set isomorphic with the fifteen cross lines of the icosahedron.

The conics on five double points cut the sextic in two other points whose parameters will be given by a quadratic in $t$. Out of a possible 252 conics, 150 are accounted for by the conics on six points. That is, 150 do not give new

\footnotetext{
* Coble, American Journal of Mathematics, vol. 46, p. 160.
} 
points in the $t$-plane. The equation of the conic on (12345) is $\left(\delta_{1}-\delta_{2}\right) x_{0} x_{2}$ $+\left(\delta_{1}^{2}-\delta_{1}{ }^{6}\right) x_{1}{ }^{2}=0$. In this equation substitute the coördinates of the sextic $S_{2}(t)$, divide by the ten-ics in $t$ which give the parameters of the points of intersection of the conic and the sextic at the five double points, and obtain as quotient a quadratic in $t$ which gives the parameters of the two remaining points of intersection. In this case the quadratic is $t_{1} t_{2}=0$ and the corresponding point in the $t$-plane is $(0,1,0)$. Under $G_{80}$ the conic (12345) goes into the five other conics (23680), (34679), (12790), (15689), and (45780). The corresponding points of these six conics in the $t$-plane are the six points which Klein calls the fundamental points. Moreover the conics on the complementary five points:

$$
\text { (67890), (14579), (12580), (34568), (23470), (12369), }
$$

give the same six points in the $t$-plane. That is, the six quadratics which give the six fundamental points with respect to $K(t)$ give two points each on $S_{2}(t)$ through which pass two conics which cut the sextic again in two different sets of five nodes. The conic on (12348) gives the point $\left(\epsilon, 3 \delta_{2},-\epsilon^{t}\right)$ with respect to $K(t)$ and is transformed into thirty conics by $G_{60}$. The conic on (56790) gives the point $\left(\epsilon, 3 \delta_{1},-\epsilon^{4}\right)$ and is transformed into thirty other conics. The conic (13567) gives the point

$$
\left[\left(3 \epsilon+7+5 \epsilon^{4}\right),-\left(4 \epsilon+4 \epsilon^{2}+2\right),\left(5 \epsilon+3 \epsilon^{4}+7\right)\right]
$$

and is transformed into thirty conics. This accounts for the 252 conics. Moreover, these points in the $t$-plane obtained from the lines on two nodes and the conics on five nodes lie on the fifteen axes conjugate under $G_{60}$.

\section{The SEXTIC OF GENUS FOUR}

Consider again the form

$$
(\pi x)(a \tau)(\alpha t)^{3}=x_{0}\left(t^{3}+3 t \tau\right)+x_{1}\left(t^{3} \tau-1\right)+x_{2}\left(3 t^{2}-\tau\right),
$$

which, when equated to zero, gives the cubic envelopes perspective to the rational sextic (2) §III. Some of the comitants given by Coble* to within a numerical factor are as follows:

(2) $\left(\pi \pi^{\prime} \xi\right)\left(a a^{\prime}\right)(\alpha t)\left(\alpha^{\prime} t\right)^{3}=\left(t_{2}{ }^{6}-3 t_{1}{ }^{5} t_{2}\right) \xi_{0}+10 t_{1}{ }^{3} t_{2}{ }^{3} \xi_{1}+\left(t_{1}{ }^{6}+3 t_{1} t_{2}{ }^{5}\right) \xi_{2}$,

(3) $(\pi x)\left(\pi^{\prime} x\right)(a \tau)\left(a^{\prime} \tau\right)\left(\alpha \alpha^{\prime}\right)^{2}(\alpha t)\left(\alpha^{\prime} t\right)=\left(x_{0} x_{1} t_{1}^{2}-x_{1} x_{2} t_{1} t_{2}-x_{0}^{2} t_{2}^{2}\right) \tau_{1}^{2}$

$+\left[x_{0}^{2} t_{1}^{2}-\left(x_{1}^{2}+2 x_{0} x_{2}\right) t_{1} t_{2}-x_{2}^{2} t_{2}^{2}\right] \tau_{1} \tau_{2}-\left(x_{2}^{2} t_{1}^{2}+x_{0} x_{1} t_{1} t_{2}+x_{1} x_{2} t_{2}^{2}\right) \tau_{2}^{2}$,

(4) $\left(\pi \pi^{\prime} \pi^{\prime \prime}\right)(a \tau)\left(a^{\prime} \tau\right)\left(a^{\prime \prime} \tau\right)\left(\alpha \alpha^{\prime}\right)\left(\alpha \alpha^{\prime \prime}\right)\left(\alpha^{\prime} \alpha^{\prime \prime}\right)(\alpha t)\left(\alpha^{\prime} t\right)\left(\alpha^{\prime \prime} t\right)=t_{1}^{8} \tau_{1}^{2} \tau_{2}+t_{1}^{2} t_{2} \tau_{2}^{8}$

$+t_{1} t_{2}^{2} \tau_{1}^{3}-t_{2}^{3} \tau_{1} \tau_{2}^{2}$.

*American Journal of Mathematics, vol. 46, p. 162. 
The cubic (4) is apolar to (1) and hence it gives the three cusps of the cubic envelope for any given $\tau$. But the cubic in (4) is the original $(3,3)$ form and hence the cusp locus will be a curve of genus four. For a cusp (1) must be a perfect cube and hence its Hessian must vanish identically. The cusp locus is then obtained by eliminating $\tau$ from the coefficients of $t_{1}^{2}, t_{1}, t_{2}, t_{2}^{2}$, in (3) which is the Hessian of (1). This gives

$$
x_{1}\left(x_{0}^{5}-x_{2}^{5}\right)+2 x_{0}^{3} x_{2}^{3}+x_{0}^{2} x_{1}^{2} x_{2}^{2}+x_{0} x_{1}^{4} x_{2}=0,
$$

the sextic of genus four invariant under the icosahedral group. Klein* states, without proof, that an infinite number of triangles whose vertices lie on (5) can be circumscribed about the conic $4 x_{0} x_{2}-x_{1}^{2}=0$ and that every point of (5) is in one such triangle. One proof is that given by Coble. $\dagger$ The theorem given there also means for this case that the lines joining the vertices to the points of contact of opposite sides intersect in a point of the rational icosahedral sextic.

Consider now the equation $G=0$ of $\S$ I. By means of the above configuration the solutions of the cubic equation $G=0$ are obtained as modular functions. The sextic (9) $\S \mathrm{I}$ is the sextic (5) where $x_{0}: x_{1}: x_{2}$ is replaced by $Y_{2}: Y_{0}:-Y_{1}$. The sextic (8) §I is also the sextic (2) §IIII. The sextic (5) in coördinates $Y$ is the locus in $S_{2}(Y)$ of the half-period points of the family of $E^{5}$ s in $S_{4}$ and is of genus four. For every $t$ there are three values of $\theta$, say $\theta$, $\theta^{\prime}, \theta^{\prime \prime}$, in $G=0$ which give tangents to the conic forming a triangle whose vertices lie on (5) and are the half-period points $u=\omega_{1} / 2, \omega_{2} / 2,\left(\omega_{1}+\omega_{2}\right) / 2$, of an $E^{5}$ in $S_{4}$. The lines joining the vertices to the points of contact of opposite sides meet in the point $u=0$ of the $E^{5}$. For a given $\omega$, a value of $t=t_{1}: t_{2}$ is obtained as $Z_{1}(0): Z_{2}(0)$ on $S_{1}(Z)$ and $t$ is determined as a function of $\omega$. The three corresponding values, $\theta, \theta^{\prime}$, and $\theta^{\prime \prime}$, which satisfy $G=0$ are obtained by means of the half-period points of the $E^{5}$. The coördinates $Y_{0}: Y_{1}: Y_{2}$ of the vertices of the triangle are obtained from

$$
Y_{0}: Y_{1}: Y_{2}=2 \theta_{1} \theta_{2}:-\theta_{1}{ }^{2}: \theta_{2}{ }^{2}
$$

as

$$
\begin{gathered}
Y_{0}\left(\frac{\omega_{1}}{2}\right): Y_{1}\left(\frac{\omega_{1}}{2}\right): Y_{2}\left(\frac{\omega_{1}}{2}\right)=\theta+\theta^{\prime}:-\theta \theta^{\prime}: 1, \\
Y_{0}\left(\frac{\omega_{2}}{2}\right): Y_{1}\left(\frac{\omega_{2}}{2}\right): Y_{2}\left(\frac{\omega_{2}}{2}\right)=\theta+\theta^{\prime \prime}:-\theta \theta^{\prime}: 1, \\
Y_{0}\left(\frac{\omega_{1}+\omega_{2}}{2}\right): Y_{1}\left(\frac{\omega_{1}+\omega_{2}}{2}\right): Y_{2}\left(\frac{\omega_{1}+\omega_{2}}{2}\right)=\theta^{\prime}+\theta^{\prime \prime}:-\theta^{\prime} \theta^{\prime}: 1 .
\end{gathered}
$$

* Mathematische Annalen, vol. 12, p. 542.

$\dagger$ American Journal of Mathematics, vol. 46, p. 179. 
Then

$$
\begin{aligned}
& \theta=\frac{1}{2}\left\{\frac{Y_{0}\left(\frac{\omega_{1}}{2}\right)}{Y_{2}\left(\frac{\omega_{1}}{2}\right)}+\frac{Y_{0}\left(\frac{\omega_{2}}{2}\right)}{Y_{2}\left(\frac{\omega_{2}}{2}\right)}-\frac{Y_{0}\left(\frac{\omega_{1}+\omega_{2}}{2}\right)}{Y_{2}\left(\frac{\omega_{1}+\omega_{2}}{2}\right)}\right\}, \\
& \theta^{\prime}=\frac{1}{2}\left\{\frac{Y_{0}\left(\frac{\omega_{1}}{2}\right)}{Y_{2}\left(\frac{\omega_{1}}{2}\right)}-\frac{Y_{0}\left(\frac{\omega_{2}}{2}\right)}{Y_{2}\left(\frac{\omega_{2}}{2}\right)}+\frac{Y_{0}\left(\frac{\omega_{1}+\omega_{2}}{2}\right)}{Y_{2}\left(\frac{\omega_{1}+\omega_{2}}{2}\right)}\right\}, \\
& \theta^{\prime \prime}=\frac{1}{2}\left\{-\frac{Y_{0}\left(\frac{\omega_{1}}{2}\right)}{Y_{2}\left(\frac{\omega_{1}}{2}\right)}+\frac{Y_{0}\left(\frac{\omega_{2}}{2}\right)}{Y_{2}\left(\frac{\omega_{2}}{2}\right)}+\frac{Y_{0}\left(\frac{\omega_{1}+\omega_{2}}{2}\right)}{Y_{2}\left(\frac{\omega_{1}+\omega_{2}}{2}\right)}\right\} \text {. }
\end{aligned}
$$

These expressions give the values of $t$ and $\theta$ satisfying $G=0$ explicitly as modular functions. As $t$ takes all values of the line $S_{1}(Z)$, all solutions of $G=0$ are obtained as modular functions.

VI. The QUARTIC SURFACES, The JACOBIAN AND THE SYMmetroId of Cayley and the Stahl quadric

The web of point quadrics apolar to $\bar{Q}_{1}+\bar{Q}_{2}$ is

(1) $z_{0}\left(2 y_{2} y_{3}-3 y_{0}^{2}\right)+z_{1}\left(2 y_{0} y_{1}+3 y_{3}^{2}\right)+z_{2}\left(2 y_{0} y_{2}+y_{1}^{2}\right)+z_{3}\left(2 y_{1} y_{3}+y_{2}^{2}\right)=0$.

Interpret $\left(y_{0}, y_{1}, y_{2}, y_{3}\right)$ as points of the space of $c_{1}(\tau)$ and $c_{2}(t)$ and $\left(z_{0}, z_{1}, z_{2}, z_{3}\right)$ as points of a second space. Then the locus of points $z$ for which (1) has a double point is

$$
\Sigma_{1}=-11 z_{0} z_{1} z_{2} z_{3}+3\left(z_{0}^{3} z_{2}-z_{1}^{3} z_{3}-z_{1} z_{2}^{3}+z_{0} z_{3}^{3}\right)+z_{0}^{2} z_{1}^{2}+z_{2}^{2} z_{3}^{2}=0,
$$

a quartic surface given by a symmetric four-row determinant whose elements are linear functions of $z$ and hence a symmetroid.

The locus of points $y$ where (1) has a double point is the Jacobian of the web:

$J_{1}=-9 y_{0} y_{1} y_{2} y_{3}+\left(-3 y_{0}^{3} y_{1}+y_{1}^{3} y_{3}+y_{0} y_{2}^{3}+3 y_{2} y_{3}^{3}\right)+9 y_{0}^{2} y_{3}^{2}-y_{1}^{2} y_{2}^{2}=0$,

a quartic surface in the space of $c_{1}(\tau)$ and $c_{2}(t)$.

The ten double points of $\Sigma_{1}$ are

$$
\left(-\delta_{j}^{2} \epsilon^{i}, \delta_{j}^{2} \epsilon^{2 i}, \epsilon^{3 i}, 1\right) \quad(i=0,1,2,3,4 ; j=1,2) .
$$


The ten lines of the Jacobian are obtained by substituting (2) in (1).

The web of quadric envelopes apolar to $Q_{1}+Q_{2}$ is

(3) $\zeta_{0}\left(2 \xi_{2} \xi_{3}-\xi_{0}^{2}\right)+\zeta_{1}\left(2 \xi_{0} \xi_{1}+\xi_{3}{ }^{2}\right)+\zeta_{2}\left(3 \xi_{1}^{2}+2 \xi_{0} \xi_{2}\right)+\zeta_{3}\left(2 \xi_{1} \xi_{3}+3 \xi_{2}^{2}\right)=0$.

For the web (3) the Cayley symmetroid becomes

$$
\Sigma_{2}=-11 \zeta_{0} \zeta_{1} \zeta_{2} \zeta_{3}+3\left(\zeta_{0}^{3} \zeta_{2}-\zeta_{1}^{8} \zeta_{3}-\zeta_{1} \zeta_{2}^{3}+\zeta_{0} \zeta_{3}^{3}\right)+\zeta_{0}^{2} \zeta_{1}^{2}+\zeta_{2}^{2} \zeta_{3}^{2}=0,
$$

and the Jacobian is

$$
J_{2}=-9 \xi_{0} \xi_{1} \xi_{2} \xi_{3}+\left(-\xi_{0}^{3} \xi_{1}+3 \xi_{1}^{3} \xi_{3}+3 \xi_{0} \xi_{2}{ }^{3}+\xi_{2} \xi_{3}^{3}\right)+\xi_{0}{ }^{2} \xi_{3}^{2}-9 \xi_{1}^{2} \xi_{2}^{2}=0 .
$$

By (1) the points of the cubic curves $c_{1}(\tau)$ and $c_{2}(t)$ map into the planes of two rational space sextic curves:

$$
\bar{R}_{1}(\tau): \quad \zeta_{0}=2 \tau-\tau^{6}, \quad \zeta_{1}=2 \tau^{5}+1, \quad \zeta_{2}=5 \tau^{4}, \quad \zeta_{3}=5 \tau^{2},
$$

and

$$
\bar{R}_{2}(t): \quad \zeta_{0}=-5 t^{4}, \quad \zeta_{1}=5 t^{2}, \quad \zeta_{2}=2 t^{5}+1, \quad \zeta_{3}=-2 t+t^{6} .
$$

The planes $\zeta$ of these curves on a point $z$ are given by the parameters of the points in which the quadrics (1) cut $c_{1}(\tau)$ and $c_{2}(t)$. The line sections of $\bar{S}_{2}(t)$ and $\bar{S}_{1}(\tau)$ give the points of intersection of $\bar{Q}_{1}$ and $\bar{Q}_{2}$ and $c_{2}(t)$ and $c_{1}(\tau)$ respectively. Since (1) is apolar to $\bar{Q}_{1}$ and $\bar{Q}_{z}$, the sextics $\bar{R}_{1}(\tau)$ and $\bar{R}_{2}(t)$ are conjugate to $\bar{S}_{1}(\tau)$ and $\bar{S}_{2}(t)$ respectively, that is, the point sections of $\bar{R}_{1}(\tau)$ are apolar to the line sections of $\bar{S}_{1}(\tau)$, and similarly for $\bar{R}_{2}(t)$ and $\bar{S}_{2}(t)$.

The point sections of $\bar{R}_{2}(t)$ may be written

$$
z_{3} t^{6}+2 z_{2} t^{5}-5 z_{0} t^{4}+5 z_{1} t^{2}-2 z_{3} t+z_{2}=0 .
$$

A line $t$ of the perspective cubic $E_{3}(t)$ cuts $\bar{S}_{2}(t)$ in a point $t$ and five other points given by (7) §IV. Set

$$
\begin{aligned}
\left(t t^{\prime}\right)\left(q t^{\prime}\right)^{5}= & \left(t_{1}^{\prime} t_{2}-t_{2}^{\prime} t_{1}\right)\left[\tau_{1}\left(-t_{1}^{\prime} t_{2}{ }^{2}-10 t_{1}^{\prime}{ }^{\prime} t_{2}^{\prime} t_{1} t_{2}-10 t_{1}^{\prime}{ }^{3} t_{2}^{\prime 2} t_{1}{ }^{2}-3 t_{2}^{\prime}{ }^{5} t_{2}{ }^{2}\right)\right. \\
& \left.+\tau_{2}\left(3 t_{1}^{\prime} t_{1}{ }^{2}-10 t_{1}^{\prime}{ }^{8} t_{2}^{\prime 2} t_{2}^{2}-10 t_{1}^{\prime} t_{2}^{\prime} t_{1} t_{2}-t_{2}^{\prime}{ }^{5} t_{1}^{2}\right)\right] .
\end{aligned}
$$

If $(4)$ is written as $(\beta z)(b t)^{6}=0$ the covariant $(\beta z)(b q)^{5}(b t)$ becomes

$$
\left(t t^{\prime}\right)(z \zeta)=\left(t_{1} t_{2}^{\prime}-t_{2} t_{1}^{\prime}\right)\left(-z_{0} t_{2} \tau_{2}+z_{1} t_{1} \tau_{1}-z_{2} t_{1} \tau_{2}+z_{3} t_{2} \tau_{1}\right),
$$

which evidently vanishes for $t=t^{\prime}$. The points $z$ on planes $\zeta$ define on $\bar{R}_{2}(t)$ a linear system of binary sextics which have a common apolar quintic. The planes $\zeta$ are then planes of the Stahl quadric.* The parametric equations of the Stahl quadric are

$$
\zeta_{0}=-t_{2} \tau_{2}, \quad \zeta_{1}=t_{1} \tau_{1}, \quad \zeta_{2}=-t_{1} \tau_{2}, \quad \zeta_{3}=t_{2} \tau_{1},
$$

* Conner, loc. cit., p. 33. 
and

$$
z_{0}=t_{1} \tau_{1}, \quad z_{1}=-t_{2} \tau_{2}, \quad z_{2}=-t_{2} \tau_{1}, \quad z_{3}=t_{1} \tau_{2},
$$

in planes and points respectively, or, in quaternary coördinates,

$$
\zeta_{0} \zeta_{1}-\zeta_{2} \zeta_{3}=0 \text { and } z_{0} z_{1}-z_{2} z_{3}=0 .
$$

Introduce the substitution

$$
5 y_{i}=\epsilon^{4 i} z_{0}-\epsilon^{i} z_{1}+\epsilon^{3 i} z_{2}+\epsilon^{2 i} z_{3} .
$$

Then

$$
5 \sum y_{i}^{2}=2\left(z_{2} z_{3}-z_{0} z_{1}\right) .
$$

The Stahl quadric must be invariant under a group of order 120 isomorphic with the symmetric group on five letters. An invariant subgroup of order 60 generated by $S$ and $T$ of (4) $\S I I$ leaves the two systems of generators invariant. An operation of period two interchanges $t$ and $\tau$ and hence the two systems of generators. The collineations of the subgroup of order sixty are

$$
\begin{aligned}
& z_{0}^{\prime}=\epsilon^{3 \mu} z_{0}, \quad z_{0}^{\prime}=-\epsilon^{2 \mu} z_{1}, \\
& S^{\mu}: \quad z_{1}^{\prime}=\epsilon^{2 \mu} z_{1}, \quad S^{\mu} U: \begin{array}{l}
z_{1}^{\prime}=-\epsilon^{3 \mu} z_{0}, \\
z_{2}^{\prime}=\epsilon^{\mu} z_{2}, \quad \epsilon^{4 \mu} z_{3},
\end{array} \\
& \text { ' } z_{3}^{\prime}=\epsilon^{4 \mu} z_{3} ; \quad z_{3}^{\prime}=\epsilon^{\mu} z_{2} \text {; } \\
& 5^{1 / 2} z_{0}^{\prime}=\epsilon^{3 \nu}\left[-\epsilon^{3 \mu} z_{0}-\epsilon^{2 \mu} z_{1}-\epsilon^{\mu} \delta_{2} z_{2}+\epsilon^{4 \mu} \delta_{1} z_{3}\right], \\
& S^{\mu} T S^{\nu}: \\
& 5^{1 / 2} z_{1}^{\prime}=\epsilon^{2 \nu}\left[-\epsilon^{3 \mu} z_{0}-\epsilon^{2 \mu} z_{1}-\epsilon^{\mu} \delta_{1} z_{2}+\epsilon^{4 \mu} \delta_{2} z_{3}\right], \\
& 5^{1 / 2} z_{2}^{\prime}=\epsilon^{\nu}\left[-\epsilon^{3 \mu} \delta_{2} z_{0}-\epsilon^{2 \mu} \delta_{1} z_{1}+\epsilon^{\mu} z_{2}-\epsilon^{4 \mu} z_{3}\right], \\
& 5^{1 / 2} z_{3}^{\prime}=\epsilon^{4 \nu}\left[\epsilon^{3 \mu} \delta_{1} z_{0}+\epsilon^{2 \mu} \delta_{2} z_{1}-\epsilon^{\mu \mu} z_{2}+\epsilon^{4 \mu} z_{3}\right] ; \\
& 5^{1 / 2} z_{0}^{\prime}=-\epsilon^{2 \nu}\left[-\epsilon^{3 \mu} z_{0}-\epsilon^{2 \mu} z_{1}-\epsilon^{\mu} \delta_{1} z_{2}+\epsilon^{4 \mu} \delta_{2} z_{3}\right], \\
& 5^{1 / 2} z_{1}^{\prime}=-\epsilon^{3 \nu}\left[-\epsilon^{3 \mu} z_{0}-\epsilon^{2 \mu} z_{1}-\epsilon^{\mu} \delta_{2} z_{2}+\epsilon^{4 \mu} \delta_{1} z_{3}\right], \\
& 5^{1 / 2} z_{2}^{\prime}=\epsilon^{4 \nu}\left[\epsilon^{3 \mu} \delta_{1} z_{0}+\epsilon^{2 \mu} \delta_{2} z_{1}-\epsilon^{\mu} z_{2}+\epsilon^{4 \mu} z_{3}\right], \\
& 5^{1 / 2} z_{3}^{\prime}=\epsilon^{\nu}\left[-\epsilon^{3 \mu} \delta_{2} z_{0}-\epsilon^{2 \mu} \delta_{1} z_{1}+\epsilon^{\mu} z_{2}-\epsilon^{4 \mu} z_{3}\right] \\
& (\mu, \nu=0,1,2,3,4) \text {. }
\end{aligned}
$$

It is more convenient to write an operation of period four which interchanges the two systems of generators. Such an operation is

$$
R: \quad z_{0}^{\prime}=z_{2}, z_{1}^{\prime}=-z_{3}, z_{2}^{\prime}=-z_{1}, z_{3}^{\prime}=z_{0},
$$

where $R^{2}=U$.

The Stahl quadric cuts the symmetroid $\Sigma_{1}$ in the curve

$$
t_{1}^{4} \tau_{1} \tau_{2}^{3}-t_{1}^{3} t_{2} \tau_{1}^{4}-3 t_{1}^{2} t_{2}^{2} \tau_{1}^{2} \tau_{2}^{2}+t_{1} t_{2}^{3} \tau_{2}^{4}-t_{2}^{4} \tau_{1}^{8} \tau_{2}=0 .
$$


The bilinear factors of (8) §IV give the double points of the symmetroid.* By means of these factors the double points of the symmetroid may be numbered to correspond with the nodes of the rational sextic:

$$
\begin{array}{ll}
1 & \left(-\epsilon^{3} \delta_{2}{ }^{2}, \epsilon \delta_{2}{ }^{2}, \epsilon^{4}, 1\right), \\
2 & \left(-\epsilon^{4} \delta_{2}{ }^{2}, \epsilon^{3} \delta_{2}{ }^{2}, \epsilon^{2}, 1\right), \\
3 & \left(-\delta_{2}^{2}, \delta_{2}{ }^{2}, 1,1\right), \\
4 & \left(-\epsilon \delta_{2}{ }^{2}, \epsilon^{2} \delta_{2}{ }^{2}, \epsilon^{3}, 1\right), \\
5 & \left(-\epsilon^{2} \delta_{2}{ }^{2}, \epsilon^{4} \delta_{2}{ }^{2}, \epsilon, 1\right), \\
6 & \left(-\delta_{1}{ }^{2}, \delta_{1}{ }^{2}, 1,1\right), \\
7 & \left(-\epsilon \delta_{1}{ }^{2}, \epsilon^{2} \delta_{1}{ }^{2}, \epsilon^{3}, 1\right), \\
8 & \left(-\epsilon^{2} \delta_{1}{ }^{2}, \epsilon^{4} \delta_{1}{ }^{2}, \epsilon, 1\right), \\
9 & \left(-\epsilon^{3} \delta_{1}{ }^{2}, \epsilon \delta_{1}{ }^{2}, \epsilon^{4}, 1\right), \\
0 & \left(-\epsilon^{4} \delta_{1}{ }^{2}, \epsilon^{3} \delta_{1}{ }^{2}, \epsilon^{2}, 1\right) .
\end{array}
$$

If six nodes of the rational sextic lie on a conic the complementary four nodes of the symmetroid lie on a plane. $\dagger$ Hence the nodes of the symmetroid lie by fours on two sets of planes, one containing ten and the other fifteen planes. The following are the sets of planes:

$\begin{array}{ll}(4790), & (1259), \\ (2780), & (3457), \\ (3689), & (1230), \\ (1679), & (1458), \\ (5680), & (2346),\end{array}$

and

$\begin{array}{lll}(3670), & (1890), & (1349), \\ (1570), & (5789), & (1247), \\ (4569), & (2379), & (2450), \\ (2690), & (4678), & (2358), \\ (3480), & (1268), & (1356) .\end{array}$

These sets of numbers also give interesting number systems. The set of ten is the arrangement of ten numbers four at a time such that each occurs four times. The set of fifteen is the arrangement of ten numbers four at a time such that each appears six times and every couple twice. These number systems as well as those which give the configuration of the nodes of the rational sextic are unaltered by the $G_{120}$ which leaves the Stahl quadric invariant.

* Coble, American Journal of Mathematics, vol. 51, p. 501.

† Coble, Algebraic Geometry and Theta Functions, 1929, p. 256. 
Corresponding to a plane section of $\Sigma_{1}$ is a curve of order six and genus three on $J_{1}{ }^{*}$ If the plane passes through four double points of $\Sigma_{1}$ the curve of order six on $J_{1}$ becomes the four lines corresponding to the four double points and the two lines which intersect these four lines. Hence corresponding to the twenty-five planes which contain four double points of the symmetroid, there are fifty additional lines on the Jacobian.

VII. The two SPACE SEXTICS AND THE CUBIC SURFACE

By (3) §VI the planes of the two space cubic curves $c_{1}(\tau)$ and $c_{2}(t)$ map into the points of two rational space sextic curves:

$$
R_{1}(\tau): z_{0}=-2 \tau^{5}-1, z_{1}=-2 \tau+\tau^{6}, z_{2}=5 \tau^{2}, \quad z_{3}=5 \tau^{4},
$$

and

$$
R_{2}(t): z_{0}=-5 t^{2}, \quad z_{1}=5 t^{4}, \quad z_{2}=t^{6}-2 t, \quad z_{3}=2 t^{5}+1 .
$$

These two sextics lie on the same cubic surface:

$$
z_{0}^{2} z_{3}+z_{1}^{2} z_{2}+z_{2}^{2} z_{0}-z_{3}^{2} z_{1}=0 .
$$

The six four-fold secants of $R_{1}(\tau)$ are the lines determined by the pencils of planes

$$
z_{2}+\lambda z_{3}=0
$$

(3) $\left[-\epsilon^{3 \mu} \delta_{2} z_{0}-\epsilon^{2 \mu} \delta_{1} z_{1}+\epsilon^{\mu} z_{2}-\epsilon^{4 \mu} z_{3}\right]+\lambda\left[\epsilon^{3 \mu} \delta_{1} z_{0}+\epsilon^{2 \mu} \delta_{2} z_{1}-\epsilon^{\mu} z_{2}+\epsilon^{4 \mu} z_{3}\right]=0$.

Moreover, $z_{2}+\lambda z_{3}=0$ cuts the sextic $R_{1}(\tau)$ in the points whose parameters are given by

$$
\tau_{1}^{2} \tau_{2}^{2}\left(\tau_{2}^{2}+\lambda \tau_{1}^{2}\right)=0 .
$$

That is, the four-fold secants are double tangents of $R_{1}(\tau)$. The six four-fold secants of $R_{2}(t)$ are also double tangents and are the lines determined by the pencils of planes

$$
z_{0}+\lambda z_{1}=0
$$

(5) $\left[-\epsilon^{3 \nu} z_{0}-\epsilon^{2 \nu} z_{1}-\epsilon^{\nu} \delta_{2} z_{2}+\epsilon^{4 \nu} \delta_{1} z_{3}\right]+\lambda\left[-\epsilon^{3 \nu} z_{0}-\epsilon^{2 \nu} z_{1}-\epsilon^{\nu} \delta_{1} z_{2}+\epsilon^{4 \nu} \delta_{2} z_{3}\right]=0$.

Conner $\dagger$ stated an opinion that the two sets of four-fold secants for the most general case would form a double six on the cubic surface. In this special case it is evidently true. $z_{2}+\lambda z_{3}=0$ does not intersect $z_{0}+\lambda z_{1}=0$ but does intersect the other five lines in (5). One of the lines (3) intersects $z_{0}+\lambda z_{1}=0$,

* Coble, American Journal of Mathematics, vol. 46, p. 191.

$\dagger$ Loc. cit., p. 42. 
intersects (5) if $\mu \neq \nu$, but does not intersect (5) if $\mu=\nu$. Similarly for the lines (4) and (5).

The Stahl quadric intersects the cubic surface (1) in the curve $F=0$.

Introduce the substitution (6) $\S \mathrm{VI}$ :

$$
3\left(z_{1}^{2} z_{2}+z_{0}^{2} z_{3}+z_{0} z_{2}^{2}-z_{1} z_{3}^{2}\right)=25 \sum y_{3}^{3} \text {. }
$$

The cubic surface (1) is the diagonal surface of Clebsch. The symmetroid satisfies the following relation:

$$
(8 / 25) \Sigma_{1}=30 \sum y_{i}^{4}-7\left(\sum y_{i}^{2}\right)^{2} .
$$

UNIVERSITY OF IIInNOIS, URBANA, InL. 